\title{
Automatic Segmentation of Retinal Nerves by Improved Fuzzy-C-Means Clustering
}

\author{
Z. Faizal Khan, Ph.D \\ Department of Computer and Network Engineering, \\ College of Engineering, Shaqra University, \\ Al Dawadmi, Kingdom of Saudi Arabia
}

\author{
Syed Usama Quadri, Ph.D \\ Department of Computer and Network Engineering \\ College of Engineering, Shaqra University, \\ Al Dawadmi, Kingdom of Saudi Arabia
}

\begin{abstract}
Computer Aided Detection of medical image has been an improved step in the early diagnosis of diseases present in the body. Developing an efficient algorithm for medical image segmentation has been a demanding area of growing research of interest during the last decades. The initial step in computer aided diagnosis of retinal medical image is generally to segment the nerves present in it. The second step is to analyze each area separately to find the presence of pathologies in it. This paper reports on segmenting of the nerves by separating the retinal images using the combination of Improved FuzzyC-Means Clustering along with the Enhanced multidimensional multiscale parser (EMMP) algorithm. The performance of this proposed approach is proved to be better for a threshold value of 120 . From the experimental results, it has been observed that the proposed segmentation approach provides better segmentation accuracy of $97.4 \%$ in segmenting Retinal nerves.
\end{abstract}

\section{Keywords}

Fuzzy-C-Means Clustering, Enhanced multidimensional multiscale parser (EMMP) algorithm, Segmentation, Retinal image.

\section{INTRODUCTION}

Segmentation methods are useful for partitioning an image into multiple segments inorder to provide an effective representation of the objects present in it. Moreover, Image segmentation is also useful for locating objects and boundaries (lines, curves, etc.) and to assign a label to every pixel in an image such that pixels with the same label share certain visual characteristics. Moreover, each of the pixels from particular regions are similar with respect to some characteristic or computed property, such as color, intensity, or texture. At the same time, the adjacent regions are significantly different with respect to the same characteristic [5].

The various regions obtained by segmenting an image can be further used for different types of analysis and interpretations. Therefore, segmentation of image involves extracting important features and deriving the relevant metrics to segregate regions of homogeneous intensities. In order to achieve this, it is necessary to choose a selective region of interest by considering the application requirements. In the past, many image segmentation methods have been proposed by various researchers for performing successive image analysis. In addition, many researchers have used the existing thresholding techniques for segmenting the various regions of interest. In short, the most frequently used techniques for segmentation are statistical methods, geometrical, structural, model based, signal processing methods, spatial domain filters, Fourier domain filtering, Gabor and wavelet models have also been used in most works present in the literature [6, 7].

The remainder of this paper is described as follows: Section 2 depicts the features and methods proposed in related works for segmenting retinal images. Section 3 explains the proposed algorithm and highlights the advantages of segmenting retinal CT images. Section 4 depicts the results. Section 5 gives the conclusion on this work.

\section{RELATED WORKS}

Accurately segmentation should be done since, the pathologies present on it may be on the boundary of the retina. Such pathologies will be lost and it reduces the detection accuracy, if the entire retinal area is not segmented accurately. Main goal of retinal region of interest segmentation is to separating the nerves corresponding to pathology region from the voxels corresponding to the surrounding anatomy.

Many techniques have been employed to the exudate detection. Gardner et al. [8] proposed an automatic detection of diabetic retinopathy using an artificial neural network. The exudates are identified from grey level images. The fundus image was analyzed using a back propagation neural network. The technique did not work well on low contrast images.

The thresholding and RRGS technique were widely used. Sinthanayothin et al. [5] reported the result of an automated detection of diabetic retinopathy on digital fundus images by RRGS algorithm where the performance was measured on $10 \times 10$ patches rather on the whole image. Usher et al. [7] detected the candidate exudates region by using a combination of RRGS and adaptive intensity thresholding. The candidate regions were extracted and used as input to a neural network. Poor quality images affected the separation result of bright and dark lesions using thresholding and exudate feature extraction using RRGS algorithm.

An effective retinal vessel segmentation technique based on supervised classification using an ensemble classifier of boosted and bagged decision trees was proposed in [16]. A novel neural network based methodology for the segmentation of retinal vessels was presented in [15]. The neural network is trained in only one database which proves to be effective and robust in segmenting the vessels present in retina images. An average accuracy of 0.9452 is obtained for the set of database images.

Zheng et al. [9] detected exudates using thresholding and a region growing algorithm. The fundus photographs were taken with a non-mydriatic fundus camera and were then scanned by a flat-bed scanner. Colour normalization and local contrast enhancement followed by fuzzy $\mathrm{C}$-means clustering and neural networks were used by Osareh et al. [10]. The system works well only on Luv colour space but in the case of 
non-uniform illumination the detection accuracy is low. Mitra et al. [11] applied naïve Bayes classifier for diagnosis of diseases from retinal image. A system can provide a good decision support to ophthalmologist.

\section{MATERIALS AND METHODS}

The proposed system is based on fuzzy clustering. In this paper, the term fuzzy clustering is used to explicit the details of the image at various depths of information. Fuzzy rules are developed and used for determining the optimum segmentation of the nerves from the background of a CT retina images. Gray scale have been taken images from retina database as each pixel can be analyzed using the proposed method. The basic block diagram of the proposed system is given in Fig. 1.

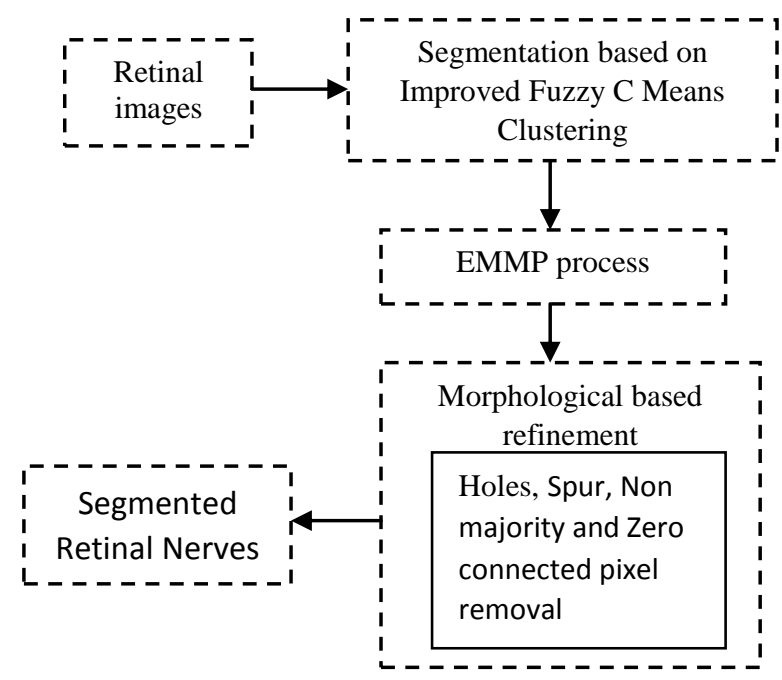

Figure 1: Block Diagram of the Proposed System

\subsection{Improved Fuzzy-C-Means Clustering}

A fuzzy similarity-based self-constructing feature clustering technique is using an incremental feature clustering method. It reduces the amount of features for the text classification approach. An improved FCM (IFCM) clustering algorithm is to split a specified set of data or objects into a cluster is the purpose of a clustering analysis, which signifies subsets or a group. The division should have two properties one of them is the homogeneity within cluster data, which belongs to on cluster, should be as same as feasible and another one is heterogeneity among the cluster data, which belongs to dissimilar clusters, should be as dissimilar as feasible [6]. Every cluster is recognized by a membership function with statistical mean value and deviation. If a pixel is not similar to any existing cluster, a new cluster is shaped for this pixel. All the pixels are desired to different group. Finally many numbers of clusters are created repeatedly and extract one feature from each and every cluster [7]. The extracted feature equivalent to a cluster is a weighted arrangement of the pixels contained in the cluster. For image segmentation, improved FCM clustering method is created by including the spatial neighborhood details into the standard FCM clustering technique by a priori probability $\left(\mathrm{p}_{\mathrm{it}}\right)$. The priori probability is set to specify the spatial weight of the neighboring pixels on the center pixel in the image. The new fuzzy membership of the current center pixel is again recalculated through this probability obtained value. The method is initialized by a known histogram based FCM algorithm [8].

Step 1: Place the cluster centroids $C_{i}$ value according to the histogram of the image, Fuzzification limitation $\mathrm{f}$, the value of $\mathrm{h}$ and $\mathrm{k}>0$

Step 2: calculate the membership function by using $u_{i g}{ }^{(a)}=\frac{1}{\sum_{\mathbf{j}=1}^{\mathbf{c}}\left[\frac{\mathbf{d}\left(\mathbf{g}, \mathbf{v}_{\mathbf{i}}\right)}{\mathbf{d}\left(\mathbf{g}, \mathbf{v}_{\mathbf{j}}\right)}\right]^{2 /(\mathbf{f}-\mathbf{1}) \forall \mathbf{i}, \mathbf{g}}}$

Step 3: Compute the cluster centroids by using

$$
\begin{gathered}
v_{i}(a+1)=\frac{\sum_{g=M_{\min }}^{M_{\max }}\left(u_{i g}^{(a)}\right)^{f} h i s(g) g}{\sum_{g=M_{\min }}^{M_{\max }}\left(u_{i g}^{(a)}\right)^{f} h i s(g)}, \forall i \\
\operatorname{His}(g)=\sum_{p=0}^{p-1} \sum_{q=0}^{q-1} \delta(l(p, q)-g)
\end{gathered}
$$

Step 4: Go to step 2 and replicate until convergence

Step 5: Calculate the a priori probability, by using

$$
p_{i t}=\frac{\nexists S_{t}^{i}}{\neq S_{t}}
$$

By obtaining the results of membership function and centroids

Step 6: Recalculate the membership function and cluster centroids by using

$$
\begin{aligned}
& u_{i t}^{*(a)}=\frac{P_{i t}}{\sum_{j=1}^{c}\left[\frac{d_{i t}}{d_{j t}}\right]^{2 /(f-1)}} \\
& v_{i}^{*(a+1)}=\frac{\sum_{t=1}^{n}\left(u_{i t}^{*(a)}\right)^{f} x_{t}}{\sum_{t=1}^{n}\left(u_{i t}^{*(a)}\right)^{f}}
\end{aligned}
$$

Step 7: If the algorithm is convergence, Go to step 8, otherwise go to step 5 .

Step 8: Image segmentation after defuzzification using

$\mathrm{C}_{\mathrm{t}}=\arg _{\mathrm{i}}\left\{\max \left(\mathrm{u}_{\mathrm{it}}\right)\right\} \mathrm{i}=1,2 \ldots, \mathrm{c}$ 
Where $\mathrm{p}_{\mathrm{it}}$ - Priori probability, $\mathrm{u}_{\mathrm{it}}$ - is the degree \& membership of $x_{t}$ in the $i^{i h}$ th cluster, C-No. of the cluster, f-weighting exponent on each fuzzy membership, $\mathrm{Ci}-$ is the prototype of the centroid of the cluster $\mathrm{i}, \mathrm{d} 2\left(\mathrm{x}_{\mathrm{t}}, \mathrm{ci}\right)$ is a distance between object $\mathrm{x}_{\mathrm{t}}$ and cluster ci,g- is a gray level.

\section{EMMP ALGORITHM}

The steps of the proposed EMMP algorithm are as follows,

Step: 1 Read the input image

Step: 2 separate the images in to number of clusters

Step: 3 Integer Wavelet Transforms is applied to convert spatial to frequency domain.

Step: 4 Quantization is done by using a global threshold

Global threshold $=120$ is set.

if (global threshold $<120)$,

Low intensity pixels. else

if (global threshold>120),

High intensity pixels

end if

Step: 8 obtain the segmented image.

\section{RESULTS AND DISCUSSION}

Accuracy of segmentation has been evaluated inorder to assess the performance of the proposed segmentation algorithm. In this section, results of the proposed methodology in terms of qualitatively and quantitatively through image display and experimental analysis respectively was present. Computed tomography Retina images of different patients taken from the Drive database have been considered in this paper. The segmented results along with its original images is given in Table 1

Table 1 Segmented Results

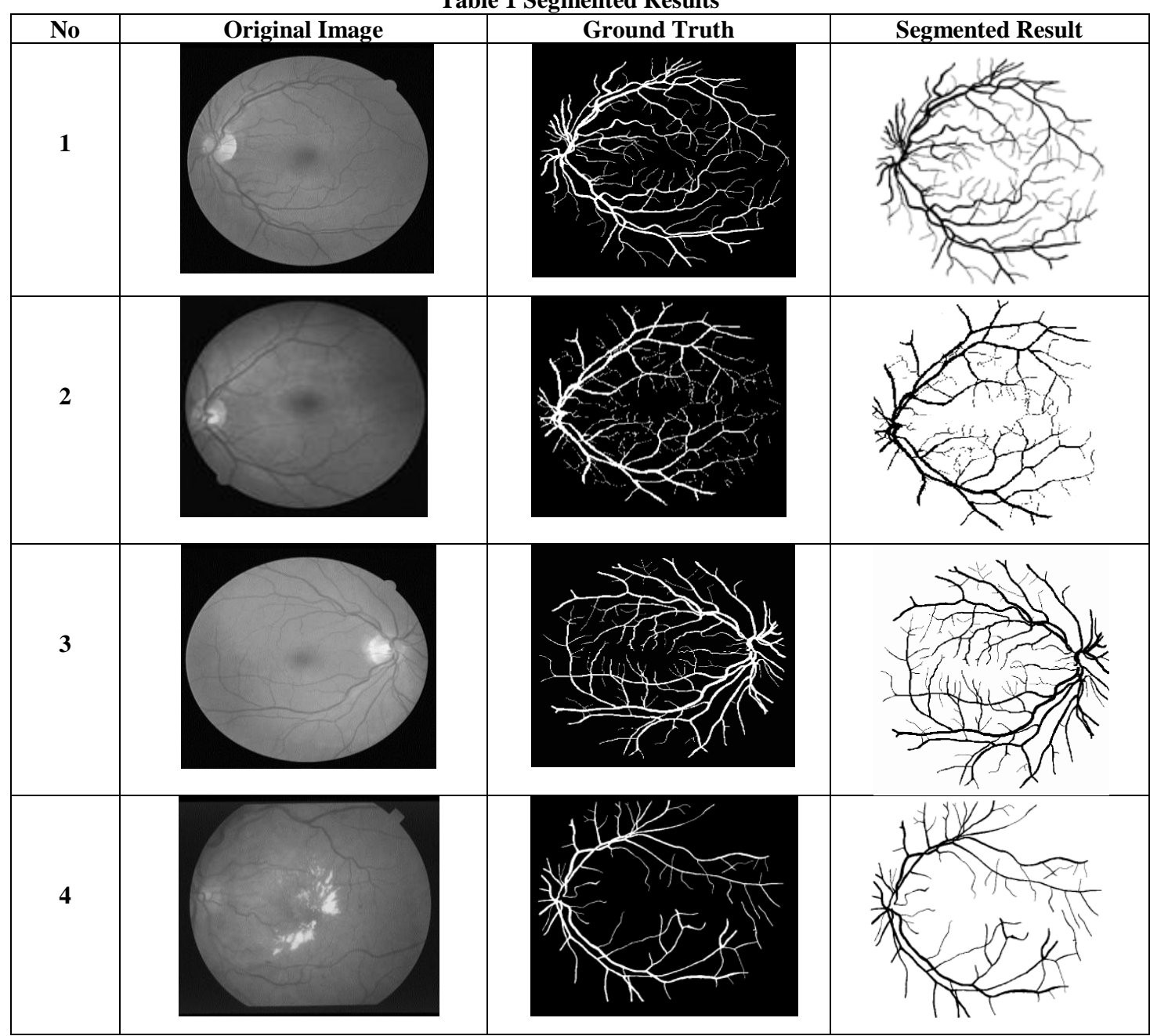

Table 1 shows the segmented images obtained from different retina images. The threshold varies from 80 to 140 . Computing the accuracy of segmentation is the common way to measure the performance of image segmentation. The objective of this comparison is to measure accuracy of the proposed segmentation algorithm based on sensitivity, specificity, and accuracy and segmentation accuracy based on number of objects. The proposed algorithm is applied to various retinal images taken from the retina database and the segmentation results are compared to the manually drawn images called ground truth. The metrics used to compare to the ground truth are sensitivity, specificity, and accuracy. Their formulas are as follows. 
Sensitivity $=\frac{T P}{T P+F N}$

Specificity $=\frac{T N}{T N+F P}$

Accuracy $=\frac{T P+T N}{T P+F N+T N+F P}$

where TP is True Positive, FP is False positive, TN is True Negative and FN is False Negative.

Table 2 Segmentation Accuracy based on Sensitivity, Specificity and Accuracy

\begin{tabular}{|l|c|c|c|}
\hline Image & Sensitivity & Specificity & Accuracy \\
\hline Image 1 & 91.4 & 98.5 & 97.1 \\
\hline Image 2 & 89.4 & 98.3 & 98.1 \\
\hline Image 3 & 89.3 & 97.3 & 97.2 \\
\hline Image 4 & 90.5 & 98.4 & 97.3 \\
\hline Average & $\mathbf{9 0 . 1}$ & $\mathbf{9 8 . 1}$ & $\mathbf{9 7 . 2}$ \\
\hline
\end{tabular}

Table 2 shows the segmentation accuracy based on Sensitivity, Specificity and Accuracy for all retina images. The performance remains closely consistent which indicates the value of the variables of the proposed algorithm are optimal. From the above table, it can be observed that our proposed segmentation method provides an overall accuracy of $97.4 \%$ in segmenting the retinal nerves effectively

\section{CONCLUSION}

In this paper a new method for segmenting retina images using the combination of Improved Fuzzy-C-Means along with the Enhanced multidimensional multiscale parser (EMMP) method had been proposed. The main purpose of this approach is to improve the accuracy of segmentation by reducing the false segmentation error. Main features of this algorithm is the use of Improved Fuzzy-C-Means Clustering along with the parser method. This method is applied to different types of retina datasets inorder to validate the efficiency of the proposed algorithm. In this proposed framework, segmentation of the normal tissues is not degraded since the unwanted section other than the region of interest is exactly removed.

\section{REFERENCES}

[1] B. S. Morse, Lecture 18: Segmentation (Region Based), 1998-2000.

[2] Giorgio De Nunzio, Eleonora Tommasi, Antonella Agrusti, Rosella Cataldo, Ivan De Mitri, Marco Favetta, Silvio Maglio, Andrea Massafra, Maurizio Quarta, "Automatic retinal Segmentation in CT Images with Accurate Handling of the Hilar Region", Journal of digital imaging, Vol 24, No 1, pp 11-27, 2011.

[3] J. Quintanilla-Dominguez, B. Ojeda-Magaña, M. G. Cortina-Januchs, R. Ruelas, A. Vega-Corona, and D. Andina, "Image segmentation by fuzzy and possibilistic clustering algorithms for the identification of microcalcifications," Sharif University of Technology
Scientia Iranica, vol. 18, pp. 580-589, 8 February 2011 2011.

[4] Sinthanayothin C, Boyce JF, Williamson TH, Cook HL, Mensah E, Lal S. Automated detection of diabetic retinopathy on digital fundus image. J Diabet Med; 19:105-12, 2002.

[5] Niemeijer M, van Ginneken B, Staal J, Suttorp-Schulten MS, Abramoff MD, Automatic detection of red lesions in digital color fundus photographs. IEEE Trans Med Imag; 24:584-92, 2005.

[6] Usher D, Dumskyj M, Himaga M, Williamson TH, Nussey S, Boyce J. Automated detection of diabetic retinopathy in digital retinal images: a tool for diabetic retinopathy screening. Diabet Med; 21:84-90, 2004

[7] Gardner GG, Keating D, Williamson TH, Elliott AT. Automatic detection of diabetic retinopathy using an artificial neural network: a screening tool. $\mathrm{Br} \mathrm{J}$ Ophthalmol; 80:940-4, 1996

[8] Zheng Liu, Opas C, Krishnan SM. Automatic image analysis of fundus photograph. In: Proceedings of the International Conference on Engineering in Medicine and Biology, vol. 2, p. 524-5, 1997.

[9] Osareh A, Mirmehdi M, Thomas B, Markham R. Automated identification of diabetic retinal exudates in digital colour images. Br J Ophthalmol; 87: 1220-3, 2003.

[10] Mitra SK, Te-Won Lee, Goldbaum M. Bayesian network based sequential inference for diagnosis of diseases from retinal images. Pattern Recogn Letters, vol.26, pp- 459470, 2005.

[11] Xizhao Wang, Yadong Wang, Lijuan Wang, "Improving fuzzy c-means clustering based on feature-weight learning", pattern recognition letters, April 2004.

[12] Mahesh Yambal1, Hitesh Gupta," Image Segmentation using Fuzzy C Means Clustering: A survey," International Journal of Advanced Research in Computer and Communication Engineering, Vol. 2, Issue 7, July 2013.

[13] Yong yang," image segmentation by fuzzy c-means clustering algorithm with a novel penalty term," computing and informatics, vol. 26, 17-31, 2007.

[14] Marin, D., Aquino, A., Gegundez-Arias, M.E., Bravo, J.M. (2011). A new supervised method for blood vessel segmentation in retinal images by using graylevel and moment invariants-based features. IEEE Transactions on Medical Imaging, 30 (1), 146-158.

[15] Fraz, M.M., Remagnino, P., Hoppe, A., Uyyanonvara, B., Rudnicka, A.R., Owen, C.G., Barman, S.A. (2012). An ensemble classification-based approach applied to retinal blood vessel segmentation. IEEE Transactions on Biomedical Engineering, 59 (9), 2538-2548. 